\title{
On Analytical Derivations of the Condition Number Distributions of Dual Non-Central Wishart Matrices
}

\author{
Michail Matthaiou, Member, IEEE, David I. Laurenson, Member, IEEE, \\ and Cheng-Xiang Wang, Senior Member, IEEE
}

\begin{abstract}
In this paper, we explore the statistical characterization of Multiple-Input Multiple-Output (MIMO) channel correlation matrices with the main focus being on their condition number statistics. More specifically, novel expressions are derived for the probability density function (PDF) and cumulative distribution function (CDF) of the MIMO condition number. Contrary to the majority of related studies, where only the common case of Rayleigh fading was considered, our investigation is extended to account for the generalized case of Ricean fading where a deterministic Line-of-Sight ( $\mathrm{LoS})$ component exists in the communication link. The overall analysis is based on the principles of random matrix theory and particularly of dual complex noncentral Wishart matrices; the latter represent a practical class of MIMO systems, namely dual-branch systems which are equipped with two transmit and receive antenna elements. All the derived formulae are validated through extensive simulations with the attained accuracy being remarkably good.
\end{abstract}

Index Terms-MIMO systems, non-central Wishart matrices, condition number, Ricean fading.

\section{INTRODUCTION}

I $\mathrm{T}$ is an indisputable fact that, nowadays, Multiple-Input Multiple-Output (MIMO) systems are considered as a hot topic in the area of wireless communications. The pioneering works of Foschini [1] and Telatar [2] demonstrated the extensive performance enhancement when multiple antenna elements are used at both ends of a radio link. While a considerable amount of research effort has been devoted to the study of MIMO technology, there are still some open aspects that have not been addressed. One of the most interesting topics is the eigenanalysis of the MIMO correlation matrix and especially the statistical determination of its condition number, commonly defined as the ratio of the largest to the smallest eigenvalue. In the MIMO context, the condition number indicates the multipath richness of the channel [3] and has also been shown to drastically affect the detection and error performance in spatial multiplexing (SM) systems [4], [5]. Hence, a detailed knowledge of the condition number statistics

Manuscript received July 2, 2008; revised October 11, 2008; accepted October 23, 2008. The associate editor coordinating the review of this letter and approving it for publication was H. Dai.

M. Matthaiou was with the Institute for Digital Communications, The University of Edinburgh, U.K. He is now with the Institute for Circuit Theory and Signal Processing, Technische Universität München, Arcistrasse 21,80333, Munich, Germany (e-mail: matthaiou@nws.ei.tum.de).

D. I. Laurenson is with the Institute for Digital Communications, Joint Research Institute for Signal and Image Processing, The University of Edinburgh, EH9 3JL, Edinburgh, U.K. (e-mail: Dave.Laurenson@ed.ac.uk).

C.-X. Wang is with the Joint Research Institute for Signal and Image Processing, School of Engineering and Physical Sciences, Heriot-Watt University, EH14 4AS, Edinburgh, U.K. (e-mail: Cheng-Xiang.Wang@hw.ac.uk).

Digital Object Identifier 10.1109/TWC.2009.080882 is highly desirable since it will offer a deeper insight into the efficient characterization of the promising MIMO technology. We point out that throughout the paper our main interest lies in dual-branch (or simply dual) MIMO configurations which are expected to be employed in the majority of future practical systems (e.g. hand-held devices), thanks to their small size and low complexity/implementation cost.

In order to conduct the above mentioned analysis, we have resorted to the use of complex Wishart matrices which have recently attracted considerable interest following the rapid development of MIMO systems. Most studies dealing with the application of Wishart matrix theory on MIMO systems elaborate on the common case of a rich scattering environment where no Line-of-Sight (LoS) component is present and the inter-element spacings are assumed to be sufficiently high; under these conditions, the entries of the channel matrix exhibit uncorrelated Rayleigh fading [1], [2], [6] and in practice we end up with the simplified case of complex central (zero-mean) Wishart matrices. The presence of a specular wavefront or a strong direct component though, violates the assumption of Rayleigh fading and the channel is said to be Ricean distributed instead. Surprisingly, despite their practical relevance, few results have been reported focusing on the eigenstatistics of Ricean channels. This fact can be attributed to the difficulty in manipulating hypergeometric functions with two matrix arguments of non-central Wishart matrices compared to the one matrix argument of central Wishart matrices [7].

With regard to the condition number statistics, we primarily recall the seminal work of Edelman [8] which revealed the vital importance of the condition number as a metric of the matrix ill-condition. However, his analysis was limited to the case of $(2 \times 2)$ central Wishart matrices with unit variance where the generalized work of Ratnarajah et al.[9] accounted for matrices of random size and with arbitrary variance. An interesting approach to model the temporal transition probabilities of the condition number using a finite-state Markov process can be found in [10]; more importantly, it was shown that the CDF of the logarithm of the condition number can be approximated particularly well via a gamma variable. Yet, all the above cited papers ([8]-[10]) were limited to Rayleighfading MIMO channels while the extension to Ricean-fading channels remains an open problem. On this basis, in the present study we explore the statistics of the condition number of a dual non-central Wishart matrix and introduce closedform formulae for its PDF and CDF as weighted sums of polynomials. For the sake of completeness, two different 
cases are assessed with the classification being based on the eigenvalues of the LoS matrix component (distinct or equal). As potential applications of our theoretical analysis, we can expect that it can facilitate the prediction of capacity and linklevel performance of MIMO channels as well as the design of space-time codes and MIMO simulations.

The remainder of the paper is organized as follows: In Section II, the fundamental properties of the theory of Wishart matrices are outlined. In Section III, new expressions for the condition number distributions are derived. In Section IV, the underlying MIMO Ricean channel model used throughout the paper is discussed followed by the numerical results as obtained by Monte-Carlo simulations. Finally, Section V concludes the paper and summarizes the key findings.

A note on notation: We use upper and lower case boldfaces to denote matrices and vectors, respectively while $\mathbb{C}$ will denote the set of complex-valued numbers. The nomenclature $\sim \mathcal{C N}(\mathbf{X}, \mathbf{Y})$ stands for a complex normally distributed matrix with mean $\mathbf{X}$ and covariance $\mathbf{Y}$. An $(n \times n)$ identity matrix is expressed as $\mathbf{I}_{n}$ while the all-zero $(n \times m)$ matrix as $\mathbf{0}_{n \times m}$. The symbols $(\cdot)^{H}$ and $(\cdot)^{-1}$ correspond to Hermitian transposition and matrix inversion whereas $\otimes$ is the Kronecker product. Finally, $\operatorname{det}(\cdot)$ and $\|\cdot\|_{F}$ respectively return the matrix determinant and Frobenius norm.

\section{Non-Central Wishart Matrices}

As was previously highlighted, we are particularly interested in dual complex non-central Wishart matrices. In such a case, a $(2 \times 2)$ complex normal random matrix $\mathbf{H}$ is considered which is distributed according to $\mathbf{H} \sim \mathcal{C N}\left(\mathbf{M}, \boldsymbol{\Sigma} \otimes \mathbf{I}_{2}\right)$. The matrix $\boldsymbol{\Sigma}=\sigma^{2} \mathbf{I}_{2}$ is the correlation matrix containing the variances $\sigma^{2}$ of the entries of $\mathbf{H}$ on its main diagonal. The so-called instantaneous MIMO correlation matrix is defined as $\widetilde{\mathbf{W}}=\mathbf{H H}^{H}$ and is said to follow the complex non-central Wishart distribution with two degrees of freedom and noncentrality matrix $\Omega=\boldsymbol{\Sigma}^{-1} \mathbf{M} \mathbf{M}^{H}$, commonly denoted as $\widetilde{\mathbf{W}} \sim \mathcal{C W}_{2}(2, \boldsymbol{\Sigma}, \boldsymbol{\Omega})^{1}$.

We now consider a scaled version of $\widetilde{\mathbf{W}}$, that is $\mathbf{W}=$ $\boldsymbol{\Sigma}^{-1} \widetilde{\mathbf{W}}$. Since $\mathbf{W}$ is a $(2 \times 2)$ Hermitian matrix, it has two real ordered eigenvalues $w_{1}>w_{2}>0$, whose joint PDF is

$f\left(w_{1}, w_{2}\right)=\exp \left[-\sum_{i=1}^{2}\left(\lambda_{i}+w_{i}\right)\right]{ }_{0} \widetilde{F}_{1}(2 ; \boldsymbol{\lambda}, \mathbf{w})\left(w_{1}-w_{2}\right)^{2}$

where $\boldsymbol{\lambda}=\left(\lambda_{1}, \lambda_{2}\right)$ contains the real ordered eigenvalues of $\boldsymbol{\Omega}$ and, in turn, $\mathbf{w}=\left(w_{1}, w_{2}\right)$; moreover, ${ }_{0} \widetilde{F}_{1}(. ; .,$.$) is the$ complex hypergeometric function of two matrix arguments [7]. A convenient version of ${ }_{0} \widetilde{F}_{1}(2 ; \boldsymbol{\lambda}, \mathbf{w})$ for the dual case was given by Gross and Richards [11] as

$$
{ }_{0} \widetilde{F}_{1}(2 ; \boldsymbol{\lambda}, \mathbf{w})=\frac{\operatorname{det}\left({ }_{0} F_{1}\left(1 ; w_{i} \lambda_{j}\right)\right)}{\left(\lambda_{1}-\lambda_{2}\right)\left(w_{1}-w_{2}\right)}
$$

with $I_{q}(\cdot)$ denoting the $q$ th order modified Bessel function of the first kind while ${ }_{0} F_{1}(s+1 ; x)$ is the classical hypergeometric function [12]

$$
{ }_{0} F_{1}(s+1 ; x)=s ! x^{-s / 2} I_{s}(2 \sqrt{x}) .
$$

\footnotetext{
${ }^{1}$ It should be noted that if $\mathbf{M}=\mathbf{0}_{2 \times 2}$ so that $\boldsymbol{\Omega}=\mathbf{0}_{2 \times 2}$, a complex central Wishart matrix is obtained, expressed as $\widetilde{\mathbf{W}} \sim \mathcal{C W}_{2}(2, \boldsymbol{\Sigma})$.
}

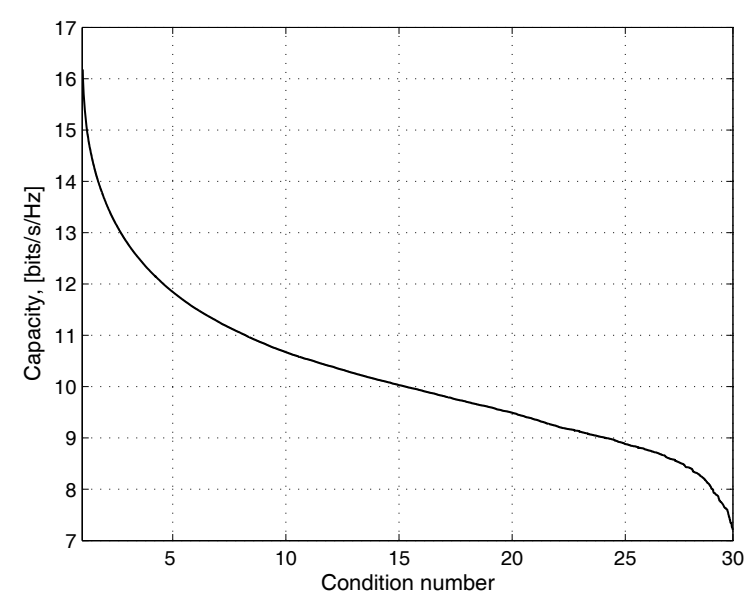

Fig. 1. Capacity evolution as a function of the condition number (SNR $=$ $20 \mathrm{~dB})$.

\section{Statistics OF THE CONDition Number OF $\mathbf{W}$}

It is well established that the condition number is a metric of the channel rank or of how invertible a given matrix is; a condition number close to one indicates a well-conditioned matrix with almost equal eigenvalues. As the condition number gets larger though, the matrix rank drops and eventually degenerates into a rank-one matrix. Its importance in the area of MIMO communications has been demonstrated in [3]-[5] among others. In the considered case, the condition number of the scaled MIMO correlation matrix $\mathbf{W}$ becomes

$$
z=\frac{w_{1}}{w_{2}} \geq 1 .
$$

From an information theory point of view, the impact of the condition number on MIMO capacity can be seen in (5), which returns the instantaneous channel capacity (in bits/s/Hz) assuming perfect channel knowledge at the Rx and no knowledge at the Tx and uniform power allocation [1]

$$
\begin{aligned}
C & =\log _{2}\left(\operatorname{det}\left(\mathbf{I}_{2}+\frac{\rho}{2} \mathbf{H} \mathbf{H}^{H}\right)\right) \\
& =\log _{2}\left(\left(1+\frac{z w_{2}}{\alpha}\right)\left(1+\frac{w_{2}}{\alpha}\right)\right)
\end{aligned}
$$

where $\rho$ is the system Signal-to-Noise ratio (SNR) and $\alpha=$ $2(1+K) / \rho$. The symbol $K$ stands for the the Ricean $K$ factor, normally expressing the ratio of the free-space signal power to the power of the scattered waves. From (5), it is evident that there is no analytical one-to-one mapping between MIMO capacity and the condition number. However, their inter-dependency can be numerically evaluated; in Fig. 1, this inter-dependency is illustrated for an SNR of $20 \mathrm{~dB}^{2}$.

This graph verifies the notion that high-rank channels, or low condition numbers, yield high capacities and vice-versa. To get a deeper understanding, the density and distribution functions of the condition number are now studied for two different cases; the distinction is based on the associated LoS eigenvalues and, in particular, on whether these are identical.

\footnotetext{
${ }^{2} \mathrm{~A}$ more detailed discussion on the simulation settings is provided in Section IV.
} 
1) Case $1\left(\lambda_{1} \neq \lambda_{2}\right)$ : We firstly consider the common case of two distinct non-zero LoS eigenvalues $\lambda_{1}>\lambda_{2}$ which reflects any conventional MIMO configuration with no constraint on the rank of the LoS channel matrix. We can show that the PDF of $z, f_{z}(z)$, can then be expressed as a weighted summation of polynomials given by

$$
\begin{aligned}
f_{z}(z)=\frac{e^{-\left(\lambda_{1}+\lambda_{2}\right)}(z-1)}{\lambda_{1}-\lambda_{2}} \sum_{k=0}^{\infty} \sum_{n=0}^{\infty} & \frac{\Gamma(k+n+3) z^{k}}{(k ! n !)^{2}(z+1)^{k+n+3}} \\
\times & {\left[\lambda_{1}^{k} \lambda_{2}^{n}-\lambda_{2}^{k} \lambda_{1}^{n}\right] }
\end{aligned}
$$

with $\Gamma(n)$ denoting the Gamma function which, for the case of an integer index, can be rewritten as $\Gamma(n)=(n-1) !$. The full proof of (6) is provided in Appendix A.

In order to reduce the high computational complexity inserted by the infinite double summation of the above equation, a truncated finite subset of terms may be considered as

$$
\begin{aligned}
f_{z}(z) \approx \frac{e^{-\left(\lambda_{1}+\lambda_{2}\right)}(z-1)}{\lambda_{1}-\lambda_{2}} \sum_{k=0}^{K_{s}} \sum_{n=0}^{N_{s}} & \frac{\Gamma(k+n+3) z^{k}}{(k ! n !)^{2}(z+1)^{k+n+3}} \\
\times & {\left[\lambda_{1}^{k} \lambda_{2}^{n}-\lambda_{2}^{k} \lambda_{1}^{n}\right] . }
\end{aligned}
$$

The values of $K_{s}$ and $N_{s}$ are chosen so that a further increase in the number of coefficients holds negligible impact on the final outcome (less than $0.5 \%$ between consecutive steps). It was empirically found that to fulfill this prerequisite with the minimum number of terms, $K_{s}$ and $N_{s}$ should be set to the same value. In fact, by adopting this approach the asymptotic result is approximated well with $K_{s}=N_{s}=20$. This observation is verified in Fig. 2, where the evolution of the double summation against the number of terms $K_{s}, N_{s}$ is depicted, for four arbitrary values of $z$.

The corresponding CDF of $z, F_{z}(x)$, can then be deduced via the PDF as

$$
F_{z}(x)=\int_{1}^{x} f_{z}(z) d z
$$

By substituting (6) into (8) and taking into account the Dominated Convergence Theorem which states that summation and integration can be interchanged, Eq. (9) at the top of the next page is obtained after some basic algebraic manipulations. For the integrals involved in (9), a tractable representation in terms of scalar hypergeometric functions is available in [12, Eq. (3.194)]

$$
\int_{0}^{u} \frac{t^{\mu}}{(1+b t)^{\nu}} d t=\frac{u^{\mu+1}}{\mu+1}{ }_{2} F_{1}(\nu, \mu+1 ; \mu+2 ;-b u)
$$

where ${ }_{2} F_{1}(\alpha, \beta ; \gamma ; u)$ is the classical Gaussian hypergeometric function defined in [12, Eq. (9.14)]. The CDF of the condition number eventually becomes

$$
\begin{gathered}
F_{z}(x)=\frac{e^{-\left(\lambda_{1}+\lambda_{2}\right)}}{\lambda_{1}-\lambda_{2}} \sum_{k=0}^{\infty} \sum_{n=0}^{\infty} \frac{\Gamma(k+n+3)}{(k ! n !)^{2}}\left[\lambda_{1}^{k} \lambda_{2}^{n}-\lambda_{2}^{k} \lambda_{1}^{n}\right] \\
\times\left\{I_{1}^{k+1, k+n+3}(x)-I_{1}^{k, k+n+3}(x)\right\}
\end{gathered}
$$

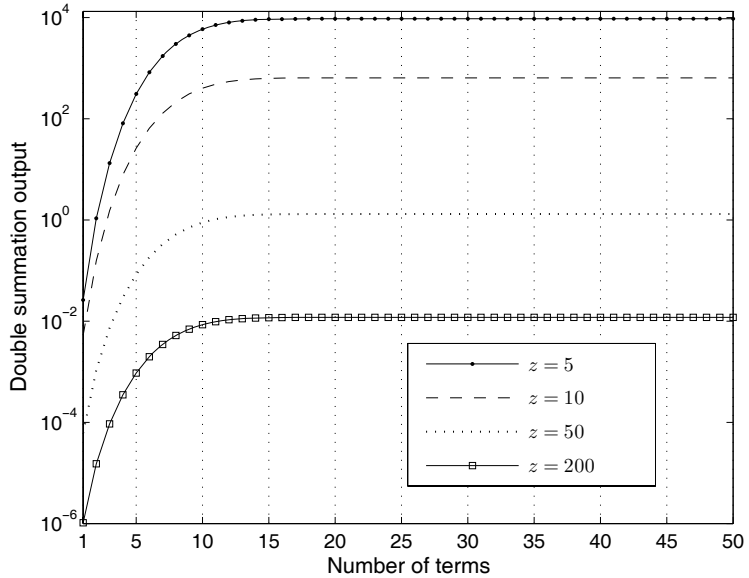

Fig. 2. Convergence of the double summation in (6) for four different values of $z$.

where

$$
\begin{aligned}
I_{1}^{a, b}(y) & =\left(\frac{y^{a+1}}{a+1}\right){ }_{2} F_{1}(b, a+1 ; a+2 ;-y) \\
& -\left(\frac{1}{a+1}\right){ }_{2} F_{1}(b, a+1 ; a+2 ;-1) .
\end{aligned}
$$

2) Case $2\left(\lambda_{1}=\lambda_{2}\right)$ : This is a special class of specifically designed full-rank LoS configurations with extensive practical interest since it offers two equal eigenvalues and thus delivers high capacities in the presence of strong direct components. This is achieved with appropriate positioning of the antenna elements at both ends of the link so that subchannel orthogonality is attained [13]-[15]. This phenomenon is contrary to the common belief that LoS channels represent a hindrance in the area of MIMO communication since they are usually rankdeficient and therefore have only one non-zero eigenvalue. In the case of equal eigenvalues though, the $\left(\lambda_{1}-\lambda_{2}\right)$ term in the denominator of (2) becomes zero making the analysis invalid; in order to circumvent this singularity we employ de l'Hôpital's rule to get a solution for the limit $\left(\lambda_{1} \rightarrow \lambda_{2}\right)$. Then, the ordered eigenvalue distribution $f\left(w_{1}, w_{2}\right)$ becomes [13]

$$
\begin{aligned}
f\left(w_{1}, w_{2}\right)= & \lambda_{1}^{-1 / 2} e^{-2 \lambda_{1}}\left(w_{1}-w_{2}\right) e^{-\left(w_{1}+w_{2}\right)} \\
\times & \left(\sqrt{w_{1}} I_{1}\left(2 \sqrt{\lambda_{1} w_{1}}\right) I_{0}\left(2 \sqrt{\lambda_{1} w_{2}}\right)\right. \\
& \left.-\sqrt{w_{2}} I_{1}\left(2 \sqrt{\lambda_{1} w_{2}}\right) I_{0}\left(2 \sqrt{\lambda_{1} w_{1}}\right)\right) .
\end{aligned}
$$

In Appendix B, it is shown that the PDF of the condition number has the following form

$$
\begin{aligned}
f_{z}(z)=e^{-2 \lambda_{1}}(z-1) \sum_{k=0}^{\infty} \sum_{n=0}^{\infty} & \frac{\Gamma(k+n+4)}{(k ! n !)^{2}(k+1)(z+1)^{k+n+4}} \\
& \times\left(z^{k+1}-z^{n}\right) .
\end{aligned}
$$

The similarity between the infinite double summations involved in (6) and (14) is apparent and hence the finite subset approximation can be used again. In this case, a similar convergence check, as the one performed for (7), revealed that the choice $K_{s}=N_{s}=15$ approximates the asymptotic 


$$
\begin{aligned}
F_{z}(x) & =\frac{e^{-\left(\lambda_{1}+\lambda_{2}\right)}}{\lambda_{1}-\lambda_{2}} \sum_{k=0}^{\infty} \sum_{n=0}^{\infty} \frac{\Gamma(k+n+3)}{(k ! n !)^{2}}\left[\lambda_{1}^{k} \lambda_{2}^{n}-\lambda_{2}^{k} \lambda_{1}^{n}\right] \\
& \times\left\{\int_{0}^{x} \frac{z^{k+1}}{(z+1)^{k+n+3}}-\int_{0}^{1} \frac{z^{k+1}}{(z+1)^{k+n+3}}-\int_{0}^{x} \frac{z^{k}}{(z+1)^{k+n+3}}+\int_{0}^{1} \frac{z^{k}}{(z+1)^{k+n+3}}\right\} .
\end{aligned}
$$

solution reasonably well. As far as the condition number CDF is concerned, the concept for deriving an analytical expression is exactly the same as in (8)-(12). Thus, it is trivial to show that for the case of equal LoS eigenvalues the condition number $\mathrm{CDF}$ is

$$
\begin{aligned}
F_{z}(x) & =e^{-2 \lambda_{1}} \sum_{k=0}^{\infty} \sum_{n=0}^{\infty} \frac{\Gamma(k+n+4)}{(k ! n !)^{2}(k+1)} \times\left\{I_{1}^{k+2, k+n+4}(x)\right. \\
& \left.-I_{1}^{n+1, k+n+4}(x)-I_{1}^{k+1, k+n+4}(x)+I_{1}^{n, k+n+4}(x)\right\} .
\end{aligned}
$$

\section{Mimo Channel Model and Numerical Results}

The underlying MIMO channel model is now presented for the case of LoS propagation. For an uncorrelated fading scenario, the channel transfer function matrix $\mathbf{H} \in \mathbb{C}^{2 \times 2}$ consists of a spatially deterministic component $\mathbf{H}_{\mathrm{L}}$ and a randomly distributed component $\mathbf{H}_{\mathrm{W}}$ which accounts for the scattered signals. Then, the channel model is

$$
\mathbf{H}=\sqrt{\frac{K}{K+1}} \mathbf{H}_{\mathrm{L}}+\sqrt{\frac{1}{K+1}} \mathbf{H}_{\mathrm{W}} .
$$

A common policy in the analysis of MIMO systems is to normalize the entries of $\mathbf{H}$ so that they have unity energy on average and the mean SNR is independent of the channel matrix. For this reason, $\mathbf{H}_{\mathrm{W}}$ is modeled as a Rayleigh distributed matrix with independent and identically distributed (i.i.d.) circular symmetric zero-mean complex Gaussian variables with unity variance. With regard to the free-space LoS component $\mathbf{H}_{\mathrm{L}}$, its entries can be expressed as $e^{-j k d_{m, n}} / d_{m, n}$, where $k=2 \pi / \lambda$ is the wavenumber corresponding to the carrier wavelength $\lambda$ and $d_{m, n}$ is the distance between a receive element $m \in\{1,2\}$ and a transmit element $n \in\{1,2\}$. Please note that we have assumed, without loss of generality, isotropic radiators. Regarding the statistical characteristics of $\mathbf{H}$, it can be inferred that $\mathbf{M}=\sqrt{\frac{K}{K+1}} \mathbf{H}_{\mathrm{L}}$ while $\boldsymbol{\Sigma}=\frac{1}{K+1} \mathbf{I}_{2}$. Then, it is trivial to show that the Wishart matrix $\widetilde{\mathbf{W}}=\mathbf{H H}^{H}$ follows the distribution $\widetilde{\mathbf{W}} \sim \mathcal{C} \mathcal{W}_{2}\left(2, \frac{1}{K+1} \mathbf{I}_{2}, K \mathbf{H}_{\mathrm{L}} \mathbf{H}_{\mathrm{L}}^{H}\right)$ and the associated $\operatorname{LoS}$ version of interest $\mathbf{W}=(K+1) \widetilde{\mathbf{W}}$.

We can now validate the theoretical analysis presented in Section III through a set of simulations. For the sake of brevity, we consider the more general case of unequal LoS eigenvalues but all the presented results are readily extensible to the case of full-rank LoS configurations. Due to space limitations, we directly consider the LoS suboptimum configuration, as originally given by the authors in [16, Eq. 20], with

$$
\mathbf{H}_{\mathrm{L}}=\left[\begin{array}{cc}
0.8384+j 0.5451 & 0.9411+j 0.3380 \\
-0.5123-j 0.8588 & 0.8384+j 0.5451
\end{array}\right] .
$$

This matrix satisfies the power constraint, i.e. $\left\|\mathbf{H}_{\mathrm{L}}\right\|_{F}^{2}=4$. After generating 50,000 random Monte-Carlo realizations of

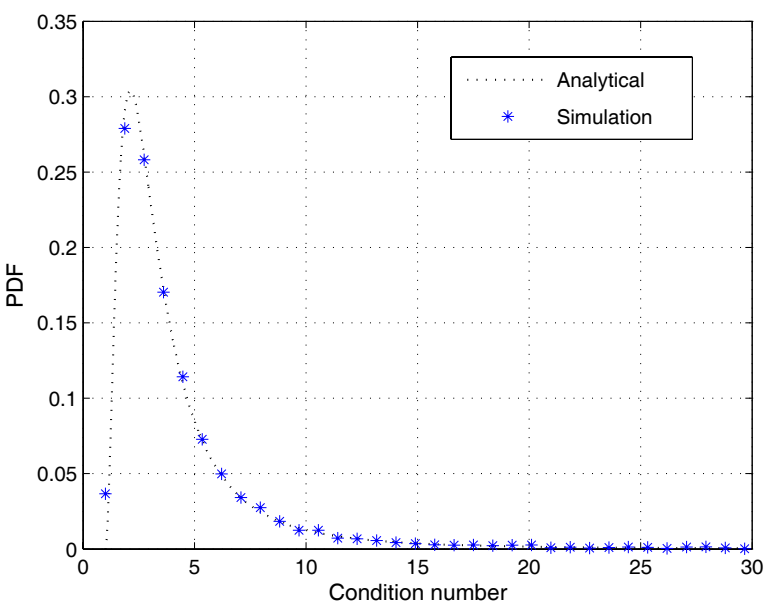

(a) PDF of the condition number.

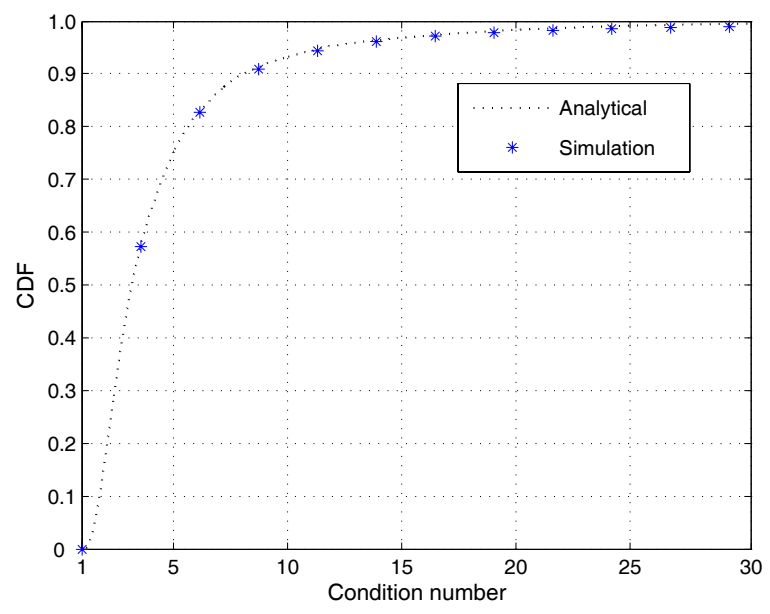

(b) $\mathrm{CDF}$ of the condition number.

Fig. 3. Condition number PDF and CDF of a dual complex non-central Wishart matrix.

the channel matrix $\mathbf{H}$ according to (16) and setting the $K$ factor equal to $5 \mathrm{~dB}$, the ordered eigenvalues of $\Omega$ were easily computed and thereafter concatenated into the vector $\boldsymbol{\lambda}=(7.0336,5.6155)$. As a main step of the performance evaluation process, the analytical expressions derived for the statistics of the condition number are validated. In Figs. 3(a) and 3(b), the closed-form relationships (6) and (11) are respectively tested, where it is easily seen that theoretical and simulation results are in remarkable agreement for both cases.

From inspection of Fig. 1 and Fig. 3, we can also conjecture that for a relatively high percentage of realizations the proposed architecture yields large capacities and outperforms 
the common i.i.d. Rayleigh system which offers an ergodic capacity of 11.4 bits $/ \mathrm{s} / \mathrm{Hz}$ for the same operating SNR. This observation is consistent with the results presented in [13][15] where different optimized architectures were proposed for the case of near-field LoS propagation.

\section{CONCLUSiON}

In the present contribution, a detailed statistical eigenanalysis of dual-branch MIMO systems has been performed. In detail, we derived the PDF and CDF of the condition number of a dual complex non-central Wishart matrix. This class of matrices has an increasing practical interest since it corresponds to compact MIMO systems with two antenna elements at both ends. The statistics of the condition number were thoroughly explored for a conventional as well as a specifically designed full-rank configuration with equal LoS eigenvalues. All theoretical formulae were tested against the outputs of a Monte-Carlo simulator and it was shown that the match between theory and simulation is excellent. It should be emphasized that all the analytical results presented in this paper can be easily evaluated since the overall complexity burden was kept as low as possible. More importantly, they constitute the basis of the statistical assessment of Ricean MIMO channels and further are very useful tools for determining numerous MIMO characteristics, spanning SM ability to symbol error and detection performance.

\section{APPENDIX A}

DERIVATION OF THE PDF OF THE CONDITION NUMBER OF

$$
\mathbf{W}\left(\lambda_{1} \neq \lambda_{2}\right)
$$

Starting with the joint eigenvalue PDF $f\left(w_{1}, w_{2}\right)$, we apply the following transformation of variables to estimate the marginal pdf of the condition number $z=w_{1} / w_{2}$

$$
f_{z}(z)=\int_{0}^{\infty} w_{2} f\left(z w_{2}, w_{2}\right) d w_{2} .
$$

By substituting (1) into (18), the integral becomes

$$
\begin{array}{r}
f_{z}(z)=e^{-\left(\lambda_{1}+\lambda_{2}\right)}(z-1)^{2} \int_{0}^{\infty} w_{2}^{3} e^{-w_{2}(z+1)} \\
{ }_{0} \widetilde{F}_{1}\left(2 ; \boldsymbol{\lambda}, \mathbf{w}^{\prime}\right) d w_{2}
\end{array}
$$

where $\mathbf{w}^{\prime}=\left(z w_{2}, w_{2}\right)$. If (2) is introduced in our analysis, the following relationship is obtained

$$
{ }_{0} \widetilde{F}_{1}\left(2 ; \boldsymbol{\lambda}, \mathbf{w}^{\prime}\right)=\frac{\operatorname{det}\left({ }_{0} F_{1}\left(1 ; w_{i}^{\prime} \lambda_{j}\right)\right)}{w_{2}\left(\lambda_{1}-\lambda_{2}\right)(z-1)}
$$

which leads to a simplified version of (19)

$$
\begin{aligned}
f_{z}(z)=\frac{e^{-\left(\lambda_{1}+\lambda_{2}\right)}(z-1)}{\lambda_{1}-\lambda_{2}} \int_{0}^{\infty} w_{2}^{2} e^{-w_{2}(z+1)} & \operatorname{det}\left({ }_{0} F_{1}\left(1 ; w_{i}^{\prime} \lambda_{j}\right)\right) d w_{2} .
\end{aligned}
$$

For the dual case, (3) reduces to ${ }_{0} F_{1}(1 ; x)=I_{0}(2 \sqrt{x})$; thus,

$$
\begin{aligned}
f_{z}(z)=\frac{e^{-\left(\lambda_{1}+\lambda_{2}\right)}(z-1)}{\lambda_{1}-\lambda_{2}} \int_{0}^{\infty} w_{2}^{2} e^{-w_{2}(z+1)} & \operatorname{det}\left(I_{0}\left(2 \sqrt{w_{i}^{\prime} \lambda_{j}}\right)\right) d w_{2} .
\end{aligned}
$$

We can now evaluate (22) as shown in (23) in the next page. To the best of our knowledge, there is no analytical solution for the integrals in (23); in this light, we express the zerothorder modified Bessel function as an infinite sum of powers according to

$$
I_{0}(x)=\sum_{k=0}^{\infty}\left(\frac{1}{k !}\right)^{2}\left(\frac{x}{2}\right)^{2 k} .
$$

Inserting (24) into (23) and taking into account the following straightforward formula

$$
\int_{0}^{\infty} x^{p} e^{-a x} d x=\frac{\Gamma(p+1)}{a^{p+1}}
$$

we conclude the proof of (6).

\section{APPENDIX B}

\section{DERIVATION OF THE PDF OF THE CONDITION NUMBER OF}

$$
\mathbf{W}\left(\lambda_{1}=\lambda_{2}\right)
$$

The derivation presented herein represents essentially an extension to the analysis of the previous section. To be more precise, by combining (13) with (18) we get (26) at the top of the next page.

Once more, the infinite series representation of a first-order modified Bessel function of the first kind is used according to

$$
\begin{aligned}
I_{1}(x) & =\frac{x}{2} \sum_{k=0}^{\infty} \frac{1}{k ! \Gamma(k+2)}\left(\frac{x}{2}\right)^{2 k} \\
& =\frac{x}{2} \sum_{k=0}^{\infty} \frac{1}{k !^{2}(k+1)}\left(\frac{x}{2}\right)^{2 k} .
\end{aligned}
$$

After substituting (24) and (27) into (26) and making use of (25), we can directly obtain (14).

\section{REFERENCES}

[1] G. J. Foschini, "Layered space-time architecture for wireless communications in a fading environment when using multiple antennas," Bell Labs Technical J., vol. 1, no. 2, pp. 41-59, Autumn 1996.

[2] I. E. Telatar, "Capacity of multi-antenna Gaussian channels," ATT-Bell Labs Internal Technical Memorandum, June 1995.

[3] V. Erceg, P. Soma, D. S. Baum, and A. J. Paulraj, "Capacity obtained from multiple-input multiple-output channel measurements in fixed wireless environments at $2.5 \mathrm{GHz}$," in Proc. International Conf. Commun. (ICC), vol. 1, New York, USA, May 2002, pp. 396-400.

[4] H. Artes, D. Seethaler, and F. Hlawatsch, "Efficient detection algorithms for MIMO channels: a geometrical approach to approximate ML detection," IEEE Trans. Signal Processing, vol. 51, no. 11, pp. 2808-2820, Nov. 2003.

[5] D. Wubben, R. Bohnke, V. Kuhn, and K. D. Kammeyer, "MMSE-based lattice-reduction for near-ML detection of MIMO systems," in Proc. ITG Workshop Smart Antennas, Munich, Germany, Mar. 2004, pp. 106-113.

[6] Ö. Oyman, R. U. Nabar, H. Bölcskei, and A. J. Paulraj, "Characterizing the statistical properties of mutual information in MIMO channels," IEEE Trans. Signal Processing, vol. 51, no. 11, pp. 2784-2795, Nov. 2003.

[7] A. T. James, "Distributions of matrix variates and latent roots derived from normal samples," Annals Mathematical Statistics, vol. 35, no. 2, pp. 475-501, June 1964.

[8] A. Edelman, "Eigenvalues and condition numbers of random matrices," Ph.D. dissertation, MIT, Cambridge, MA, May 1989.

[9] T. Ratnarajah, R. Vaillancourt, and M. Alvo, "Eigenvalues and condition numbers of complex random matrices," SIAM J. Matrix Analysis Applications, vol. 26, no. 2, pp. 441-456, Jan. 2005.

[10] P.-H. Kuo, P. J. Smith, and L. M. Garth, "A Markov model for MIMO channel condition number with application to dual-mode antenna selection," in Proc. Veh. Technol. Conf. (VTC), Dublin, Ireland, Apr. 2007, pp. 471-475.

[11] K. I. Gross and D. S. Richards, "Total positivity, spherical series, and hypergeometric functions of matrix argument," J. Approximation Theory, vol. 59, no. 2, pp. 224-246, 1989. 


$$
\begin{aligned}
f_{z}(z)=\frac{e^{-\left(\lambda_{1}+\lambda_{2}\right)}(z-1)}{\lambda_{1}-\lambda_{2}} \times & {\left[\int_{0}^{\infty} w_{2}^{2} e^{-w_{2}(z+1)} I_{0}\left(2 \sqrt{z w_{2} \lambda_{1}}\right) I_{0}\left(2 \sqrt{w_{2} \lambda_{2}}\right) d w_{2}\right.} \\
& \left.-\int_{0}^{\infty} w_{2}^{2} e^{-w_{2}(z+1)} I_{0}\left(2 \sqrt{z w_{2} \lambda_{2}}\right) I_{0}\left(2 \sqrt{w_{2} \lambda_{1}}\right) d w_{2}\right] . \\
f_{z}(z)=\lambda_{1}^{-1 / 2} e^{-2 \lambda_{1}}(z-1) \times & {\left[\int_{0}^{\infty} \sqrt{z} w_{2}^{5 / 2} e^{-w_{2}(z+1)} I_{1}\left(2 \sqrt{z \lambda_{1} w_{2}}\right) I_{0}\left(2 \sqrt{\lambda_{1} w_{2}}\right) d w_{2}\right.} \\
& \left.-\int_{0}^{\infty} w_{2}^{5 / 2} e^{-w_{2}(z+1)} I_{1}\left(2 \sqrt{\lambda_{1} w_{2}}\right) I_{0}\left(2 \sqrt{z \lambda_{1} w_{2}}\right) d w_{2}\right] .
\end{aligned}
$$

[12] I. S. Gradshteyn and I. M. Ryzhik, Table of Integrals, Series, and Products, 6th ed. San Diego: Academic Press, 2000.

[13] F. Bøhagen, P. Orten, G. E. Øien, and S. de la Kethulle de Ryhove, "Exact capacity expressions for dual-branch Ricean MIMO systems," IEEE Trans. Commun., vol. 56, no. 12, pp. 2214-2222, Dec. 2008.

[14] F. Bøhagen, P. Orten, and G. E. Øien, "Design of capacity-optimal highrank line-of-sight MIMO channels," IEEE Trans. Wireless Commun., vol. 6, no. 4, pp. 1420-1425, Apr. 2007.

[15] M. Matthaiou, D. I. Laurenson, and C. -X. Wang, "Capacity study of vehicle-to-roadside MIMO channels with a line-of-sight component," in Proc. Wireless Commun. Networking Conf. (WCNC), Las Vegas, USA, Mar. 2008, pp. 775-779.

[16] - , "Reduced complexity detection for Ricean MIMO channels based on condition number thresholding," in Proc. International Wireless Commun. Mobile Computing Conf. (IWCMC), Crete, Greece, Aug. 2008, pp. 988-993. 